\title{
Tecnologias para a Conscientização e Redução do Desperdício de Água e seu Uso de Forma Consciente: Uma Revisão Sistemática da Literatura
}

\author{
Paul Symon Ribeiro Rocha ${ }^{1}$, Nicolas Oliveira Melo ${ }^{1}$, Leonardo dos Santos ${ }^{1}$, Selma \\ Ramos Sousa ${ }^{1}$ \\ ${ }^{1}$ Instituto Federal de Educação, Ciência e Tecnologia do Maranhão (IFMA) \\ Barreirinhas - MA - Brasil \\ paul.rocha@ifma.edu.br, \{nicolasoliveiramelo3, leonsantosio01, \\ selmaramossousa\} @gmail. com
}

\begin{abstract}
Water is a universal good and because of this fact, life prevails. However, it is known that this resource is threatened. Therefore, the objective of the current paper is to provide a Systematic Review of Literature, in which an analysis of scientific works regarding to technological tools used with the purpose of reducing and raising awareness about the excessive water waste was carried out. Specifically in the following databases: REB, CBIE, WIE, SBIE, RBIE, RENOTE, JAIE, DesafIE, RITA and WAVE2. The search resulted in 22 chosen works in the preliminary selection phase, 8 of these works were included for data extraction, presenting tools and their application methodology, related to the theme area study.
\end{abstract}

Resumo. A água é um bem universal e graças a ela a vida prevalece. Porém, sabe-se que esse recurso se encontra ameaçado. Logo, o objetivo deste artigo é apresentar uma Revisão Sistemática da Literatura, onde foi realizada uma análise em trabalhos científicos a respeito de ferramentas tecnológicas utilizadas com o propósito de diminuir e conscientizar sobre o gasto demasiado de água. Especificamente nas seguintes bases de dados: REB, CBIE, WIE, SBIE, RBIE, RENOTE, JAIE, DesafIE, RITA e WAVE2. A busca resultou em 22 trabalhos selecionados na fase de seleção preliminar, onde 8 desses trabalhos foram incluídos para extração de dados, apresentando ferramentas e a metodologia de sua aplicação, dentro da temática do estudo.

\section{Introdução}

O crescente aumento da população urbana no mundo desencadeia uma forma desenfreada no gasto dos recursos hídricos. A falta de organização e planejamento neste modelo prevalece até a atualidade, tornando a infraestrutura e saneamento básico ofertados precários. A importância da água na vida dos seres humanos é imprescindível. Desde o surgimento das primeiras civilizações, aglomerados de pessoas se instalaram às margens de rios ou em costas marítimas. Os povos antigos desenvolveram maneiras de lidar com os recursos hídricos. Porém, com o desenvolvimento da agricultura surge os primeiros problemas ecológicos causados pelo sedentarismo humano [Casarin 2018].

Problemas como a escassez estão relacionados ao desperdício de água no mundo, e graves consequências são decorrentes deste fato. Segundo dados da [PNUD/RDH 2006] 
cerca de 1,1 bilhão de pessoas não possuem acesso à água tratada em todo o mundo; aproximadamente 2,6 bilhões de pessoas não têm acesso ao saneamento; apontando um alto índice de desigualdade distributiva da água no mundo.

No âmbito educacional, a água é um fator fundamental a ser trabalhado com o intuito de gerar consciência e promover hábitos para a prevenção. Segundo [Pedroza 2017] a água é um tema oportuno na promoção de ação dos sujeitos, compreensão e tomada de decisões, com relação as mudanças da sociedade e do ambiente à sua volta.

Afirmando que a água pode ser considerada base fundamental no tema transversal denominado Educação Ambiental.

Diante das inovações e dos avanços das tecnologias digitais ao longo do tempo, usar estas ferramentas no processo de combate ao desperdício e conscientização da preservação dos recursos hídricos pode ser de significativa importância. De acordo com [Rocha 2018], em decorrência do crescente avanço tecnológico, atualmente os estudantes tem acesso a uma grande quantidade de informações ofertadas pelo mundo digital, onde a popularização da internet, e a democratização das tecnologias, promove a oportunidade aos usuários, de aprender, construir, compartilhar e disseminar conhecimentos.

Dentro destes aspectos, este trabalho tem como alvo a confecção de uma Revisão Sistemática da Literatura, analisando publicações que apresentem como tema, tecnologias e metodologias tecnológicas para o uso sustentável da água. Este trabalho apresenta artigos da literatura brasileira, e suas maneiras de combater o desperdício e promover conscientização quanto ao uso racional da água, através de ferramentas tecnológicas digitais.

A Revisão Sistemática trata-se de um mecanismo utilizado no mapeamento de trabalhos publicados voltados para um tema específico, dando ao pesquisador a possibilidade de analisar o conhecimento existente sobre aquele assunto [Biolchini et al 2007]. Consistindo em um levantamento metodológico de estudos primários, selecionando uma tecnologia por meio de identificação, avaliação e interpretação de resultados alcançados através de procedimentos pré-definidos de coleta de dados [Kitchenham 2004].

\section{Fundamentação Teórica}

Neste tópico da fundamentação teórica serão abordados os seguintes temas: água e tecnologias.

\section{1 Água}

Um estudo realizado pela [Ana 2019] mostra que são utilizados no Brasil em média 2 milhões e 85 mil litros de água por segundo. Décadas atrás, esse valor era de aproximadamente $6,3 \%$ do total. Mais da Metade desse valor de consumo (52\%) é utilizado no setor de irrigação, $23 \%$ no abastecimento urbano, $9,1 \%$ é gasto pela indústria, $8 \%$ na criação de animais, $3,8 \%$ é direcionada as termelétricas, $1,7 \%$ de todo consumo abastece a zona rural e 1,6\% é destinado para o setor de minério. Dessa forma é possível perceber que o setor de irrigação em conjunto com o de abastecimento urbano, gera um consumo de 1 milhão 563 mil e 750 litros de água por segundo.

O uso de água tem aumentado em uma taxa de $1 \%$ ao ano desde a década de 80 , a demanda por água no mundo continuará crescendo até o ano de 2050, visto que com o aumento populacional e as grandes demandas dos países emergentes, o consumo será correspondente a uma taxa de $20 \%$ e $30 \%$ em relação ao nível atual [Koncagül 2019]. 
O relatório afirma que setores como o de irrigação e pecuária são os maiores consumidores de água, correspondendo a $69 \%$ de toda água gasta no planeta, visto que aproximadamente $80 \%$ de todas as terras utilizadas para agricultura no planeta utilizam a irrigação, a indústria por sua vez junto com o setor de produção de energia responde por $19 \%$ do consumo mundial e as residências populares por 12\% [Koncagül 2019]. [Lopes 2017], afirma que a conscientização sobre a gestão dos recursos hídricos é destacada como um elemento de formação na Educação Básica. Neste cenário as inovações tecnológicas surgem como um meio estratégico de sustentar e viabilizar o gasto excessivo de água, que ao ser incorporado às tecnologias de produção gera uma economia nos recursos e na racionalidade do consumo.

\subsection{Tecnologias}

As perspectivas sobre uma futura escassez de água potável têm aumentado junto com o crescimento populacional, o uso excessivo de água e a poluição dos mananciais são alguns exemplos de fatores que contribuem para essa situação. O consumo racional é uma das soluções para a preservação desses recursos naturais, e isso significa a implantação de novas medidas tecnológicas para o uso eficiente e sem desperdício [Lombardi 2012]. As tecnologias digitais tornaram-se uma aliada importante no processo educacional de um pensamento consciente, crítico a respeito do uso racional dos recursos hídricos na atualidade [Lopes 2017].

A poluição de rios e lençóis freáticos tem forte contribuição no custo da água distribuída a população, pois, as companhias de saneamento básico cobram caro no tratamento desses recursos. O consumo demasiado da água também é uma preocupação governamental e com isso algumas medidas foram criadas pelo governo bem como programas e leis que instituíram diretrizes para o uso racional da água [Lombardi 2012].

Segundo [Lombardi 2012], por meio de estudos em edificações é possível medir e conhecer os equipamentos responsáveis, pelo maior consumo de água, em uma residência e através desse conhecimento novo, tecnologias podem ser aplicadas para diminuir o consumo, bem como os arejadores de tonteira, redutores de pressão, bacias sanitárias com adicionamento duplo e torneiras com fechamento automático, os resultados são significativos e permitem a redução de até $70 \%$ do consumo.

Diversas ferramentas são desenvolvidas com o propósito de reduzir e conscientizar a população sobre o uso adequado dos recursos hídricos. Os dispositivos móveis que antes eram usados principalmente ou exclusivamente para fazer ligações, e que é visto por muitos como forma de diversão, tornou-se uma ferramenta fundamental para envolver as pessoas em temáticas importantes e relevantes, desenvolvendo um pensamento crítico [Diniz 2016].

\section{Metodologia}

O planejamento desta Revisão Sistemática da Literatura, foi realizada de acordo com o modelo de protocolo estabelecido por [Biolchini et al. 2007] e por [Kitchenham 2004], conforme apresentado nas subseções a seguir. Foram seguidas as seguintes etapas para alcançar os objetivos desta pesquisa:

Etapa 1: Definição dos objetivos e das questões de pesquisa; Etapa 2: Definição da estratégia de busca e seleção dos estudos primários; Etapa 3: Definição dos critérios de inclusão e exclusão; Etapa 4: Definição de critérios e procedimentos para seleção de estudos; Etapa 5: Definição do processo de seleção preliminar; Etapa 6: Definição do 
processo de seleção final; Etapa 7: Definição do formulário de extração dos resultados; Etapa 8: Construção das strings de busca; Etapa 9: Aplicação das strings de busca e obtenção dos trabalhos; Etapa 10: Seleção preliminar dos trabalhos; Etapa 11: Seleção final dos trabalhos; Etapa 12: Extração dos resultados e por fim, Etapa 13: Análise dos dados e consolidação dos resultados.

\subsection{Objetivos da pesquisa}

Os objetivos de pesquisa desta revisão sistemática da literatura são:

Objetivo 1: Compreender o atual estado da literatura sobre o estudo a respeito do uso de ferramentas tecnológicas para conscientizar e diminuir o desperdício de água.

Objetivo 2: Identificar quais ferramentas tecnológicas que são utilizadas na atualidade para atenuar e repensar sobre o desperdício de água.

Objetivo 3: Descrever os métodos utilizados por essas ferramentas na conscientização e redução do desperdício de água.

Objetivo 4: Entender a eficácia do uso destas ferramentas a fim de conscientizar a respeito da diminuição do desperdício de água

\subsection{Questões de Pesquisa}

A seguir são apresentadas as questões de pesquisa desenvolvidas para a Revisão Sistemática, baseadas nos objetivos citados na subseção 3.1.

Questão de pesquisa 01: Quais ferramentas tecnológicas são utilizadas na atualidade para atenuar e repensar quanto ao desperdício de água?

Questão de pesquisa 02: Quais os métodos utilizados por essas ferramentas na conscientização e redução do desperdício de água?

Questão de pesquisa 3: Qual a eficácia do uso destas ferramentas na conscientização e diminuição do desperdício de água?

\subsection{Estratégia de busca}

A estratégia de busca e seleção dos estudos primários foi definida de acordo com as fontes de estudos, palavras-chave, string de busca, critérios de inclusão e exclusão, selecionados para a revisão:

Critério de seleção das fontes: As fontes utilizadas para essa revisão sistemática foram bibliotecas digitais, cujos artigos nelas contidos eram acessíveis. Além disso, essas bibliotecas permitiam consulta online por meio de um mecanismo de busca no qual era possível utilizar expressões lógicas para definir a string de busca.

Métodos de busca de fontes: As fontes foram acessadas via mecanismos de busca das bibliotecas digitais.

Palavras-chave: Água, software, programa, aplicativo, app, tecnologia, technology, sistema, rede social, robótica, jogo, dispositivo, dispositivos, móvel, informática, gamification, gamificação, ferramenta.

String de busca: ("agua" OR "água" OR "water") AND (software OR programa OR aplicativo OR app OR tecnologia OR technology OR sistema OR rede social OR robótica OR jogo OR dispositivo OR dispositivos OR móvel OR informática OR gamification OR gamificação OR ferramenta). 
Foram utilizadas 10 fontes de pesquisa, sendo elas: 1-REB: Revista Eletrônica de Biologia, 2-Congresso Brasileiro de Informática na Educação (CBIE), 3-Workshop de Informática na Escola (WIE), 4-Simpósio Brasileiro de Informática na Educação (SBIE), 5-Revista Brasileira de Informática na Educação (RBIE), 6-Revista Novas Tecnologias na Educação (RENOTE), 7-JAIE - Jornada de Atualização em Informática na Educação, 8-DesafIE - Workshop de Desafios da Computação Aplicada à Educação, 9-Revista de Informática Teórica e Aplicada (RITA), E 10-Workshop on Advanced Virtual Environments and Education. (WAVE2)

\subsection{Critérios e Procedimentos para Seleção de Estudos}

Nesse tópico são apresentados os critérios de inclusão e os critérios de exclusão, assim como os procedimentos de seleção preliminar e seleção final dos estudos.

\subsubsection{Critérios de Inclusão}

Critério de inclusão 01: Artigos que apresentem soluções tecnológicas para conscientização e/ou redução do gasto excessivo de água;

Critério de inclusão 02: Trabalhos que apresentem como essas ferramentas foram aplicadas para conscientizar e/ou solucionar o desperdício de água.

\subsubsection{Critérios de Exclusão}

Critérios de exclusão 01: Artigos que não se encaixam nos objetivos propostos nesta RSL;

Critérios de exclusão 02: Artigos que não façam o uso de alguma tecnologia ou similares para conscientização e/ou redução do gasto excessivo de água;

Critérios de exclusão 03: Trabalhos não completos (resumos expandidos, etc.).

\subsection{Processo de Seleção}

\subsubsection{Processo de Seleção dos Estudos Primários (Processo de seleção preliminar)}

Para o processo de seleção preliminar, a string de busca foi submetida nas fontes de busca relacionadas. Os trabalhos recuperados foram inicialmente armazenados. Em seguida realizou-se a leitura dos títulos e resumos desses trabalhos. Caso existisse alguma dúvida em relação ao trabalho, a introdução e a conclusão foram lidas.

Constatando-se a relevância de um trabalho, ele era selecionado para ser lido na íntegra. Em seguida, documentou-se e selecionou-se com base nos critérios previamente definidos.

\subsubsection{Processo de seleção final}

O processo de seleção final consistiu na leitura completa dos trabalhos selecionados na etapa de seleção preliminar. Fez-se uma síntese geral e algumas considerações sobre os resultados observados nos trabalhos selecionados e definiu-se a aceitação para extração dos resultados com base nos critérios de inclusão.

\subsubsection{Estratégias de Extração e Sumarização dos Resultados}

A estratégia de extração de informação adotada foi que para cada estudo selecionado por meio dos critérios de inclusão, um formulário de coleta de dados seria preenchido, contendo as suas respectivas respostas para as questões de pesquisa. Quanto à sumarização dos resultados, os artigos encontrados durante a revisão, foram agrupados através de informações contendo tanto sínteses discursivas quanto tabuladas.

\subsubsection{Números obtidos.}


Nesta subseção são apresentados os resultados obtidos com a condução da revisão sistemática, de acordo com os objetivos propostos. Ressalta-se que após a leitura dos 22 trabalhos selecionados na fase de seleção preliminar, foi avaliada a qualidade de cada um deles em relação as questões de pesquisa. Cabe enfatizar que 2 não estavam disponíveis para visualização na fonte REB, sendo, portanto, automaticamente excluídos. Oito desses trabalhos foram incluídos para extração de dados e 14 foram excluídos por não estarem de acordo com os objetivos e as questões de pesquisa deste estudo.

Tabela 1. Quantidade de trabalhos encontrados e incluídos.

\begin{tabular}{|l|l|l|}
\hline Fonte & Total de artigos & Artigos incluídos \\
\hline REB & 8 & 0 \\
\hline CBIE & 3 & 3 \\
\hline WIE & 1 & 1 \\
\hline SBIE & 4 & 1 \\
\hline RBIE & 0 & 0 \\
\hline RENOTE & 5 & 3 \\
\hline JAIE & 0 & 0 \\
\hline DesafIE & 0 & 0 \\
\hline RITA & 1 & 0 \\
\hline WAVE2 & 0 & 0 \\
\hline TOTAL & 22 & 8 \\
\hline
\end{tabular}

\section{Resultados e discussões}

Esta seção apresenta as respostas das questões de pesquisa definidas através do protocolo pré-estabelecido nesta Revisão Sistemática da Literatura.

5.1. Questão de pesquisa 01: Quais ferramentas tecnológicas são utilizadas na atualidade para atenuar e repensar quanto ao desperdício de água?

Dentre os 8 trabalhos selecionados nesta revisão sistemática, 5 deles utilizam a gamificação como objeto de aprendizagem, conscientizando os usuários de forma não convencional sobre o desperdício e preservação dos recursos hídricos. Os trabalhos que abordam esse tipo de ferramentas são: [Diniz 2016]; [Lopes 2017]; [Oliveira 2016]; [Albuquerque 2008]; [Pedroza 2017]. Os 3 trabalhos restantes, utilizam outras ferramentas, sendo um deles uma plataforma de ensino para educação a distância [Albuquerque, 2008]; um aplicativo para smartphone [Oliveira 2019]; e um Sistema de Irrigação Inteligente (SII) [Reinoso 2017]. A maior parte das ferramentas apresentadas nos trabalhos selecionados foram projetadas para o uso em dispositivos móveis.

\subsection{Questão de pesquisa 02: Quais os métodos utilizados por essas ferramentas na} conscientização e redução do desperdício de água?

Em [Oliveira 2016], a partir do cadastro de 88 estudantes dos ensinos fundamental e médio, durante um mês de utilização da rede social, foi aplicado um questionário com 36 estudantes dos 88 cadastrados. O formulário apresenta diferentes níveis de objetivo de 
uso da ferramenta, como por exemplo: conscientizar, interagir ou curiosidades. Maior parte dos estudantes presentes na pesquisa, responderam que o objetivo do uso da ferramenta foi a conscientização.

No estudo de [Albuquerque 2008], foi realizado com alunos do curso de Ciências Biológicas da Faculdade de Ciências Biológicas e da Saúde da Universidade Iguaçu, tendo como amostra, alunos do sexto período cursando a disciplina de Zoologia. Utilizando de entrevistas, questionários para obtenção de dados após o uso do ambiente $\mathrm{EAD}$, preenchimento de formulários e o laboratório de informática.

O estudo de [Albuquerque 2008], desenvolveu e utilizou jogos como: "Planeta Água", "limpe o Rio", "Decifre o Enigma", "Quebra-cabeça", com o intuito de fixar conteúdos de educação, principalmente relacionados ao uso racional da água. Logo após foram aplicados questionários e entrevistas para a obtenção de resultados.

A pesquisa em [Pedroza 2017], foi realizada com 80 alunos dos cursos de Licenciatura em Ciências da Natureza e Química da Universidade Federal de Integração Latino-americana, de Foz do Iguaçu - PR/Brasil. Sendo desenvolvida uma sequência didática a partir de discussões dentro da abordagem CTSA, com o intuito de fortalecer a temática água. Foi iniciada uma discussão sobre a temática água, para depois os alunos terem contato com os jogos, e então avaliarem a proposta didática em questão. O primeiro jogo utilizado, foi o Dino's Adventure, abordando temas sobre o ciclo da água. O segundo jogo foi o Water Nymph, trazendo como enredo assuntos sobre Educação Ambiental, sustentabilidade e Participação Social.

No estudo de [Oliveira 2019], a partir da ação pedagógica da escola estadual Melo Viana, viu -se a necessidade de se construir um aplicativo que conscientizasse a população de Monte Carmelo-MG sobre o consumo consciente de água, através da construção colaborativa de 10 denúncias anônimas de desperdício de água. Dessa forma foram levantados requisitos, sendo seis requisitos funcionais (RF), e cinco requisitos não funcionais (RNF). Com os requisitos estabelecidos, foram realizadas reuniões para modelagem de dados do software.

Em [Diniz 2016], foi realizada uma pesquisa na loja virtual Play Store, disponível em dispositivos móveis para Android. Nessa busca constataram-se cinco aplicativo voltados para o uso da água, dos quais quatro nacionais e um internacional. A pesquisa tem como público alvo a comunidade acadêmica da unidade que é composta de servidores, colaboradores e alunos. Quanto ao público discente, o estudo é trabalhado com os alunos das graduações de dois dos polos presenciais do interior do estado de Pernambuco. Tratando-se do EcoÁgua, será promovido o aprendizado de consumo consciente de água por meio de aplicativo para dispositivos móveis fazendo uso da aprendizagem móvel (m-learning), uma vez que o aplicativo traz telas com dicas de consumo consciente e permite que o usuário ponha em prática ações de consumo responsável.

No estudo de [Lopes 2017], a caixa de areia foi aplicada com estudantes do sexto ano do ensino fundamental de uma escola pública municipal participantes do projeto interdisciplinar de Tecnologias Digitais do Programa de Bolsas de Iniciação à Docência (PIBID), da cidade de Blumenau (SC) e do projeto Defesa Civil na Escola do mesmo município. Os estudantes da escola municipal participaram três vezes da atividade e os da defesa civil uma vez. A avaliação efetiva da atividade foi feita pelos professores que acompanharam as turmas considerando a motivação dos estudantes. Na atividade os 
estudantes foram divididos em 12 grupos em função do espaço limitado em torno da caixa de areia. Portanto, as respostas analisadas referem-se aos grupos.

Em [Reinoso 2017], o SII foi desenvolvido por quatro estudantes em quatro dias. Estes estudantes possuem idades entre 14 e 17 anos, e cursam o ensino médio integrado, dois deles são alunos do curso técnico em administração e os outros do curso técnico em arquitetura. Nenhum deles possuía nenhuma experiência anterior em atividades de programação ou mesmo utilizando softwares que requerem instruções por linha de comando. Antes de iniciarem a manipulação e construção dos equipamentos reais, os estudantes construíram um protótipo virtual por meio do ambiente interativo e reativo Autodesk Circuits ${ }^{\circledR}$, que permite projetar e construir simulações de projetos eletrônicos.

\subsection{Questão de pesquisa 3: Qual a eficácia do uso destas ferramentas na conscientização e diminuição do desperdício de água?}

Em [Oliveira 2016], foi possível constatar que mais de 50\% dos alunos se sentiram motivados a utilizar a água de forma consciente, após o uso da rede social.

No estudo de [Albuquerque 2008], em um gráfico apresentado no artigo, é possível notar unanimidade entre os alunos, quando questionados se o sistema oferece informações e conhecimento necessário para compreensão do tema. Afirmando que após o uso do ambiente, vão promover ações para o uso consciente da água.

Em [Albuquerque 2008] houve uma mudança no comportamento dos alunos quanto aos temas relacionados à necessidade de preservação do meio ambiente.

O estudo de [Pedroza 2017], apresenta a interface dos jogos, e os resultados em forma de gráfico, podendo constatar que os jogos podem ser potenciais estimuladores no aprendizado quanto aos temas relacionados à água.

Em [Oliveira 2019], a ideia é que o aplicativo não seja encarado do ponto de vista de punição aos infratores, mas, pelo contrário, de ensino e difusão das consequências do desperdício de água para a comunidade local e global por meio de notícias relacionadas com as categorias das denúncias. Além de poder ser utilizado dentro de sala de aula, também poderá atuar como uma ferramenta gratuita para a comunidade local, dado que os estudantes poderão atuar como influenciadores de seus familiares e pessoas próximas.

No estudo de [Diniz 2016], o uso do EcoÁgua, pretende incentivar o consumo consciente da água por parte dos envolvidos na pesquisa. Imagina-se que o usuário ao visualizar e comparar o seu gasto hídrico com o de outros usuários, bem como com a média de consumo brasileira e do país que mais consome tenha uma noção sobre seu consumo e/ou desperdício.

Em [Lopes 2017], o artigo apresentou uma das contribuições da Realidade Aumentada para o projeto caixa de areia interativa, o qual já foi utilizado por instituições como a Defesa Civil e escolas participantes do PIBID. O uso da Realidade Aumentada em dispositivo móvel possibilitou um experimento prático para observar e discutir a motivação proporcionada pelos novos recursos digitais que estão mais próximos dos interesses das gerações mais jovens e explorou os benefícios de trabalhar com materiais concretos (reais) combinados com objetos virtuais.

No estudo de [Reinoso 2017], o sensor de chuva funciona como um medidor de resistência, conforme o nível de água no sensor aumenta a resistência diminui e assim ele pode indicar se está chovendo. Caso o nível de chuva indicado pelo sensor seja suficiente 
para garantir um nível de umidade necessária no solo, o sistema mantém a bomba de água desligada, evitando desperdício de água e energia, assim como desgaste do equipamento. O SII garante que o sistema de irrigação apenas seja ativado caso exista necessidade, que seja utilizada uma vazão adequada de água, e que a bomba de água fique apenas pelo tempo necessário.

\section{Conclusão}

Este trabalho apresentou o planejamento, a condução e os resultados de uma Revisão Sistemática da Literatura sobre tecnologias para a redução do desperdício de água e seu uso de forma consciente, com trabalhos publicados nos últimos anos, principalmente em âmbito nacional. Em particular, nas seguintes revistas: REB, CBIE, WIE, SBIE, RBIE, RENOTE, JAIE, DesafIE, RITA e WAVE2. Ressalta-se que foi realizada uma busca por artigos, o qual resultou em 22 trabalhos selecionados na fase de seleção preliminar, onde 8 desses trabalhos foram incluídos para extração de dados.

Tendo em vista as questões de pesquisa, foi possível apresentar ferramentas tecnológicas utilizadas para reduzir o gasto excessivo dos recursos hídricos e conscientizar sobre a importância dessa redução.

Além disso, os resultados obtidos forneceram os métodos desenvolvidos por cada uma dessas ferramentas para conscientizar e combater o desperdício de água, tornando essas ferramentas recursos didáticos, dinamizando o ensino-aprendizagem de alunos. Dessa forma, o desenvolvimento dessas ferramentas proporcionou um incentivo consciente, fazendo com que cada usuário, independentemente de sua faixa etária, coloque em prática ações de consumo sustentável dos recursos hídricos presentes no ambiente.

\section{Referências}

Albuquerque, R. C., \& Leite, S. Q. M. (2008). Uso de ambientes virtuais de aprendizagem como estratégia educacional complementar de ensino de ciências. RENOTE-Revista Novas Tecnologias na Educação, 6(2).

Albuquerque, R. C., Miranda, A. C., \& Kneipp, R. E. (2008). Promovendo o ensinoaprendizagem de educação ambiental no ensino fundamental com jogos baseados em ferramentas computacionais. RENOTE-Revista Novas Tecnologias na Educação, $6(2)$.

Ana, Agência Nacional de Águas. Manual de Usos Consuntivos da Água no Brasil. Brasília: ANA, 2019.

Biolchini, J.; Mian, P.; Natali, A.; Conte, T. E Travassos, G. Scientific research ontology to support systematic review in software engineering. Advanced Engineering Informatics, v. 21, n. 2, p. 133-151, 2007.

Casarin, Fátima; Dos Santos, Mônica. Água: o ouro azul: Usos e abusos dos recursos hídricos. Editora Garamond, 2018.

Diniz, Juliana; Ferreira, Andreza; Da Silva Junior, José Edmilson Gomes. EcoAgua: mlearning e gamification como estratégias de suporte ao desenvolvimento do consumo sustentável de água. In: Anais dos Workshops do Congresso Brasileiro de Informática na Educação. 2016. p. 847.

Kitchenham, Barbara. Procedures for performing systematic reviews. Keele, UK, Keele University, v. 33, n. 2004, p. 1-26, 2004. 
Koncagül, E. et al. Relatório Mundial das Nações Unidas sobre o Desenvolvimento dos Recursos Hídricos 2019. Programa Mundial das Nações Unidas para Avaliação do Recurso Hídricos Gabinete do Programa de Avaliação Global da Água. Divisão de Ciências da Água. 2019.

Lombardi, Lucas Ruiz. Dispositivos poupadores de água em um sistema predial: análise da viabilidade técnico-econômica de implementação no instituto de pesquisas hidráulicas. Porto Alegre: Trabalho de Diplomação de graduação em Engenharia Civil,2012.

Lopes, Mauricio et al. Caixa de areia interativa: um jogo em realidade aumentada no dispositivo móvel sobre a água. In: Anais dos Workshops do Congresso Brasileiro de Informática na Educação. 2017. p. 524.

Oliveira, A., Oliveira, G., Silva, A., Dionísio, M., Barreiros, E., \& França, R. (2016, November). Processo de Desenvolvimento e Avaliação de uma Rede Social Gamificada para conscientização acerca do consumo da Água. In Anais do Workshop de Informática na Escola (Vol. 22, No. 1, p. 933)

Oliveira, Danilo; Carvalho, Vitor; Araújo, Rafael. SOSÁGUA-MC: um aplicativo colaborativo para promoção do consumo consciente de água e apoio à educação ambiental. In: Anais dos Workshops do Congresso Brasileiro de Informática na Educação. 2019. p. 769.

Pedroza, Thayanna; Ferreira, Thiago; Das Graças Cleophas, Maria.

Desenvolvimento e avaliação de Jogos Educativos Digitais (JED) sobre a temática água: um estudo de caso utilizando a ferramenta GameMaker: Studio. RENOTE- Revista Novas Tecnologias na Educação, v. 15, n. 2, 2017.

PNUD/RDH. Relatório de desenvolvimento humano: além da escassez: poder, pobreza e a crise mundial da água. PNUD/ONU, 2006.

Reinoso, Luiz et al. Robótica experimental com uma arquitetura pedagógica para montagem de um sistema de irrigação inteligente. In: Brazilian Symposium on Computers in Education (Simpósio Brasileiro de Informática na Educação-SBIE). 2017. p. 695.

Rocha, Juliana Corrêa Taques et al. TIC NO ENSINO-APRENDIZAGEM DO CICLO DA ÁGUA: UMA PROPOSTA TRANSVERSAL NO ENSINO MÉDIO. RENOTERevista Novas Tecnologias na Educação, v. 16, n. 1, 2018. 